\title{
HACBA: New Approach to Hierarchical Routing for Wireless Sensor Network
}

\author{
L. T. M. Zoby \\ Electrical Engineering \\ Department of Electrical \\ Engineering, Faculty of \\ Technology, University of \\ Brasília, Brazil.
}

\author{
M. P. Carvalho \\ Electrical Engineering \\ Department of Electrical \\ Engineering, Faculty of \\ Technology, University of \\ Brasília, Brazil.
}

\author{
L. B. Ruiz \\ Computer Science \\ Department of Computer \\ Science, University of Maringá, \\ Brazil.
}

\author{
J. C. da Costa \\ Electrical Engineering \\ Department of Electrical \\ Engineering, Faculty of \\ Technology, University of \\ Brasília, Brazil.
}

\begin{abstract}
Wireless Sensor Networks are composed by sensors distributed in an environment. They are responsible to monitor and transmit its physical characteristics. These networks require robust wireless communication protocols that are energy efficient. In this paper, we present a hierarchical protocol, Hardware-Adaptative Clustering Based Algorithm (HACBA), derived from the Low Energy Adaptive Clustering Hierarchy protocol (LEACH). The protocol was applied to forest fire detection and takes into account some importants parameters of the hardware avaiable to the application chosen. This last characteristic is the main difference between the HACBA and the other protocols existing in the literature.
\end{abstract}

\section{Keywords}

Wireless Sensors Network, protocol architecture

\section{INTRODUCTION}

Wireless Sensor Networks (WSNs) are considered one of the most important technologies in the 21st century [1] [2]. Communication networks are characterized by the presence of sensor nodes that cooperate on an ad hoc basis. The objective is to monitor different environments enabling fault diagnosis on a machine [3], military supervision of a war zone [4] [5], health monitoring [6], transportation [7], industry [8] [9] and monitoring of forest fires [10] [11], among others.

One of the main challenges in a WSN application is the energy restriction [4] [7]. Other challenges include minimizing response time and providing comprehensive and reliable information [7]. To overcome these limitations, a WSN must have an efficient routing protocol for operation management.

There are many routing protocols in the literature. They are basically divided into flat routing protocols and hierarchical routing [4]. In the former, all sensor nodes have the same task. In the second, they have different levels of hierarchy between sensor nodes.

The hierarchical protocol are established two distinct nodes: sensor nodes and cluster heads $(\mathrm{CH})$. A process called clustering, that will be explained latter, forms different groups of nodes. Within each group, there is a node responsible for communication management termed $\mathrm{CH}$. The $\mathrm{CH}$ receives data from all other nodes in the cluster and sends them to the base station, which is responsible for managing all the system data. These data undergo reduction or aggregation in the $\mathrm{CH}$ in order to reduce the number of transmitted messages to the base station.

In this paper, we present a hierarchical protocol, HardwareAdaptative Clustering Based Algorithm (HACBA), derived from the LEACH (Low Energy Adaptive Clustering Hierarchy) protocol [3]. The HACBA is based on different routing protocols in the literature. The main goal is to improve the information exchange process in a WSN. For this reason, the focus was to develop an optimum code capable of reducing energy consumption and improving the quality of the sent messages. The great advantage is that it takes into account the hardware's characteristics. Thus the protocol adapts the specific characteristics of the sensor node to do the routing.

The HACBA protocol proposed is generic, it can be applied to several WSN applications. In this work this protocol was developed for forest fire detection in Brazil.

Fires cause loss of forest resources, impoverishment of the genetic heritage and natural ecosystems. The efficient and agile detection and monitoring of fire are critical and can be performed by the WSNs.

In the following section, an overview of related works to the protocol model is presented. Section III describes the protocol's characteristics in order to understand its operation. Section IV presents the communication protocols used to send messages from one node to another (CSMA, TDMA, etc.). Section $\mathrm{V}$ shows the first comparative simulation between the HACBA protocol and the LEACH. Section VI presents the result analysis. Finally, in Section VII, the conclusions are summarized.

\section{BACKGROUND}

The WSN are formed by a set of devices called sensor nodes. They combine processing power and wireless communication. Each sensor node can be viewed as an autonomous computational entity [10].

A WSN can be characterized by the use of a large number of nodes equipped with sensors, embedded microprocessors, memory and radio transceivers, capable of acquiring and 
processing signals and wireless communication. These nodes are deployed in a region of interest or close to it [12].

The main features of sensor networks can be separated into five groups of activities, as proposed in [13]: network establishment, maintenance, sensing, processing and communication. These phases may be simultaneous in their occurrence and can be active at different times of the sensor networks' lifetime.

One of the challenges in the WSN design is the low power consumption requirement [14]. Systems on chip have proven being effective in this kind of implementation. They provide energy saving and adequate performance [15], minimizing of the time response and they enable comprehensive and reliable information [16]. As previously mentioned, WSNs have routing protocols to manage theirs operation.

\section{HACBA PROTOCOL'S ARCHITECTURE}

As stated earlier, the new protocol was adapted from the LEACH protocol, but its conception considers the main ideas of other routing protocols.

The HACBA protocol simulates the node distance and the communication radius parameter providing criteria for the sensor node selection (commercial platform or custom-made node).

The sleeping mode concept presented in [17] was developed to treat redundancy [18], i.e., nodes that have the same or very similar information. In the sleeping mode some nodes around an active node are kept off, in order to save energy and also eventually to avoid the deliverance of redundant information and the consequent energy waste.

Another strategy for energy consumption optimization is the data analysis by the $\mathrm{CH}$ before sending them to the base station, such as QoS-based [16]. For instance, in this case, if a given parameter is under the alert level the information would not be sent to the base station. The $\mathrm{CH}$ is responsible for the processing. The $\mathrm{CH}$ sends the message with the lowest parameter and the sensor node IP.

Initially, it is assumed that all sensor nodes know their positions. This is due to embedded systems, such as GPS or other location systems [19] [20] [21] [22]. The sensor nodes are also capable to determine their distances to other nodes and to the base station, through the use of algorithms such as AMEER (Adaptive Minimum Energy Relay Routing) [16].

The sensor nodes have two important features related to the clustering process: communication range and sensing radius. The former concerns the maximum distance at which two sensor nodes can exchange data. The latter is related to the maximum distance from a given sensor node that an event can be sensed.

Each sensor node is part of a vector space. Different data, such as position in the $\mathrm{x}$ and $\mathrm{y}$ axes, temperature, humidity, energy, type of node (advanced or normal node or cluster head, see section V) are stored within the protocl's struct.

The $\mathrm{CH}$ choice is made when the vector is processed with the restriction that another $\mathrm{CH}$ does not exist within the area limited by the communication radius.

The new approach provided by HACBA is that the node distance between two neighbor nodes is taken into account to avoid information redundancy and to save energy. In addition, the $\mathrm{CH}$ are chosen once and forever avoiding energy waste with additional election steps. This optimization increases the system's lifetime as presented in Section V.

\section{COMMUNICATION PROTOCOLS}

After establishing the routing logic, the data transmission protocols are defined. Existing protocols in the literature were used in this paper.

Once the the $\mathrm{CH}$ are chosen, the clusters must be created. The $\mathrm{CH}$ sends a advice message (ADV - advice) using the Media Access Control (MAC) non-persistent Carrier Sensing Multiple Access (CSMA) [23]. This way the channel is tested to verify if it is free to send the data. If busy, the station waits an aleatory growing time before testing if the channel is free again. If free, the data is sent. The ADV contains the cluster head's IP and a header indicating that this is an advice message.

The sensor nodes that receive the message are located within the $\mathrm{CH}$ communication radius. Therefore they can identifiy the clump to which they belong.

In the reply task the sensor nodes sends a join-request message (Join REQ) back to the $\mathrm{CH}$, using the non-persistent CSMA MAC protocol, to inform that it will become parts of the cluster.

Once the cluster formation is concluded, the data transmission begins. In this case, the Time Division Multiple Access (TDMA) protocol is applied. Each node must wait his turn to send the signal in order to avoid data collision and unnecessary energy use.

The same protocol is used for sending data from the $\mathrm{CH}$ to the base station.

\section{PROTOCOL SIMULATION AND COMPARISON TO LEACH}

\subsection{Protocol Simulation Parameters}

The considered scenario: forest area subject to combustion events and monitored by a WSN previously randomly deployed and activated. The simulation was carried out using the parameters described in Table 1.

Table 1. Simulation Parameter

\begin{tabular}{|l|l|}
\hline \multicolumn{1}{|c|}{ Parameter } & \multicolumn{1}{c|}{ Value } \\
\hline Number of simulation rounds & 10,000 \\
\hline Sensing area (square meters) & $100 \times 100$ \\
\hline Grid (square meters) & $1 \times 1$ \\
\hline Sensor node number & 100 \\
\hline Advanced nodes & 10 \\
\hline Sensing radius (meters) & 10 \\
\hline Communication radius (meters) & $(15$ and 70$)$ \\
\hline Data Rate (Mb/s) & 1 \\
\hline Message length (bytes) & 500 \\
\hline Header length (bytes) & 25 \\
\hline
\end{tabular}


Some nodes were considered as having more energy. Those nodes were named advanced sensor nodes.

The transfer rate, the message length, the header length values can be altered in the struct according to the hardware's characteristics.

In the HACBA protocol, the simple dissipation energy model and the data reception model are used as proposed by [3]. The free-space and multipath channel models are taken into account depending on the transmitter and receiver distance [3].

\subsection{Node Deployment and Clustering representation}

All the main elements that compose a WSN are presented in this work, according to the following symbology (see Figure $1)$.

Border of the area under fire indicating the sensor nodes there positioned;
$O$ - Sensor nodes
十 - Advanced nodes;
$x$ - Base Station;
or ${ }^{*}$-Cluster heads;
- Sensor node without power; and
$\otimes$ - Sleeping node.

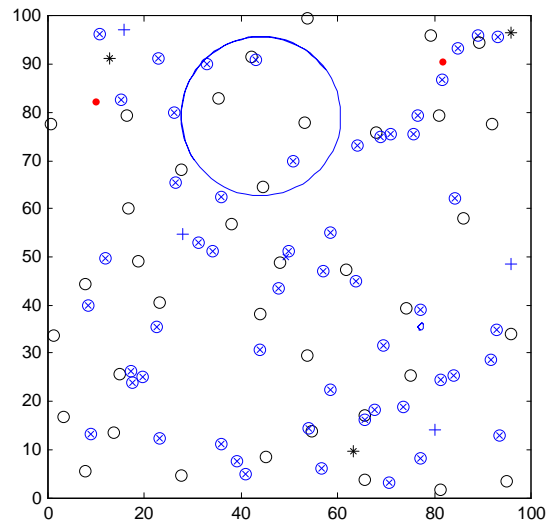

Fig. 1 HACBA protocol

\section{RESULTS AND DISCUSSION}

The node distribution in LEACH is presented in Figure 2. As previously mentioned, LEACH does not analyse the hardware's characteristics such as sensing and communication radiuses. Figure 2 shows different $\mathrm{CHs}$ near each other and the redundancy of sensor node distribution which compromises the protocol efficiency.

The simulation results, presented in Figures 3 and 4, indicate that an increase of the communication radius reduces the number of CHs. The use of a lower communication radius results in a higher amount of information sent to the base station. In this case, an eventual increase of the analysis delay can occur because each $\mathrm{CH}$ sends the information at a given time slot.

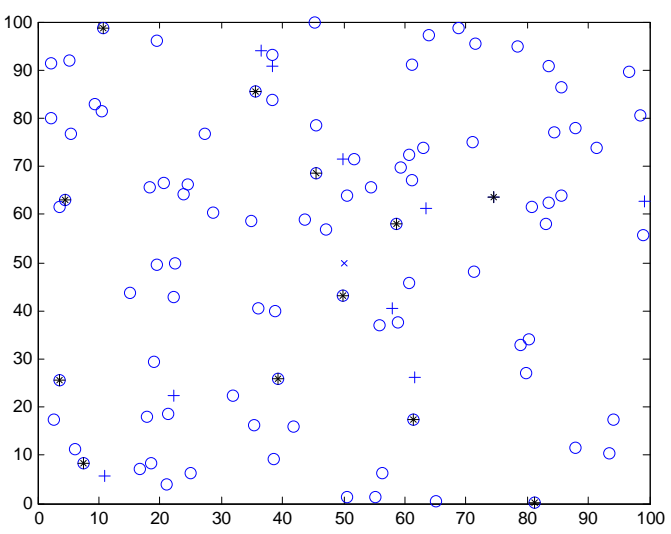

Fig 2: CH attribution by the LEACH protocol

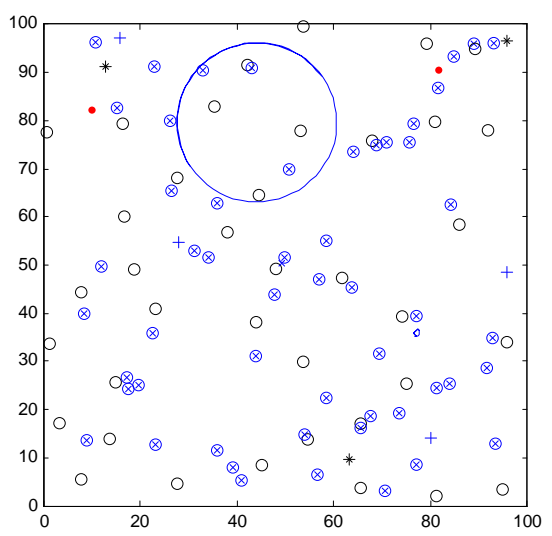

Fig. $3 \mathrm{CH}$ attribution by the HACBA protocol $(\mathrm{rc}=70 \mathrm{~m})$

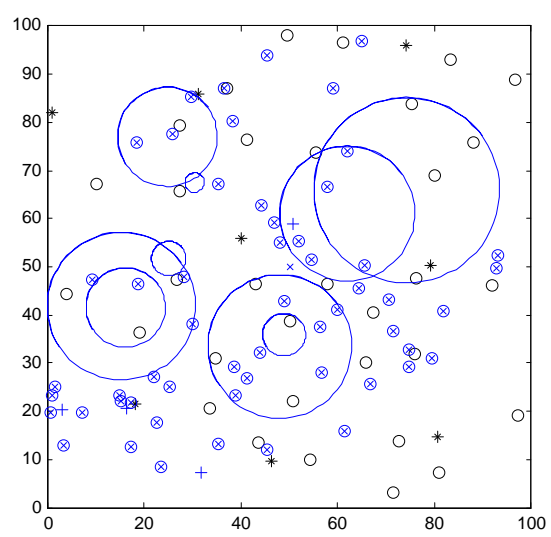

Fig. $4 \mathrm{CH}$ attribution by the HACBA protocol $(\mathrm{rc}=15 \mathrm{~m})$ 
Figure 5 shows the number of packages sent to the base. LEACH presents an average value of 10 sent packages, an inconstancy which is related to cluster dynamic formation. This inconstancy is due to the great quantity of messages sent by the $\mathrm{CHs}$ per round. The clustering process is repeated every round thus modifying constantly the number of $\mathrm{CHs}$. On the other hand, HACBA is more constant because the clustering process is less frequent: a $\mathrm{CH}$ must die before the next process of choice happens.

The results presented in Figure 5 also indicate that HACBA15 produces a higher number of $\mathrm{CH}$ increasing the number of packages sent to the base station and the energy consumption compared to HACBA-70.

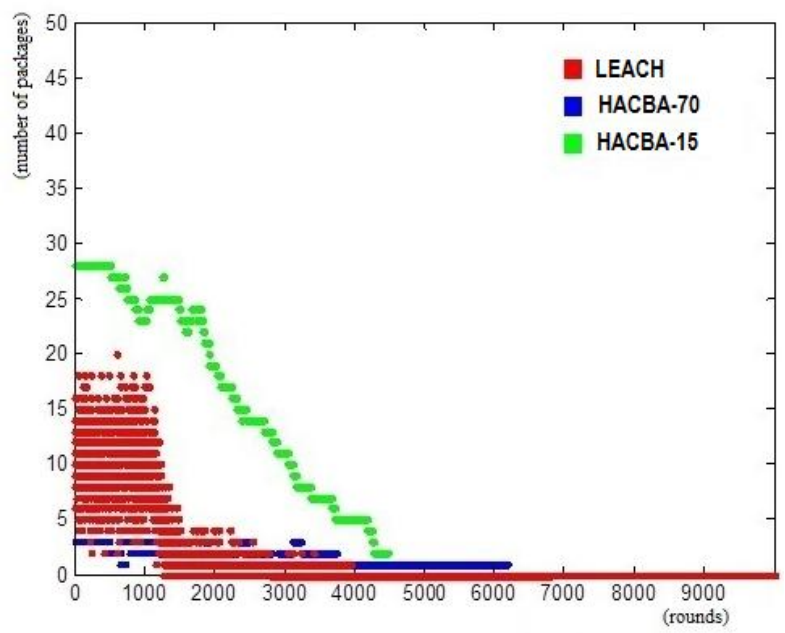

Fig. 5 Number of packages sent to the base station against rounds

The average energy and the number of dead nodes in a simulation round are presented in Figures 6 and 7.

The two simulations with the HACBA indicate the death of the last node after round 4000. In the LEACH, this occurs close to round 2000, Figure 7. The node death can be related to the lack of energy presented in Figure 6. The reduction of $\mathrm{CH}$ numbers also resulted in energy use optimization, thus increasing the lifetime of the sensor nodes.

The comparison between the HACBA and the LEACH indicates that the former presents higher efficacy.

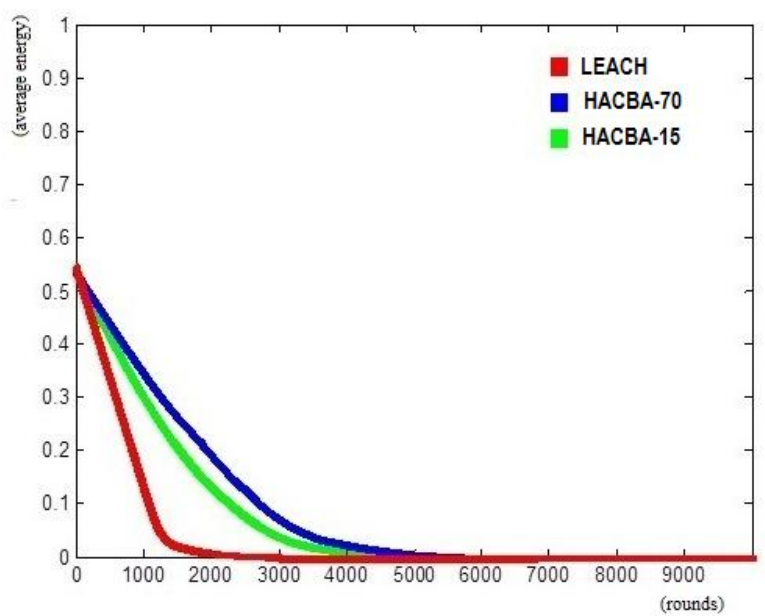

Fig. 6 Average energy against rounds

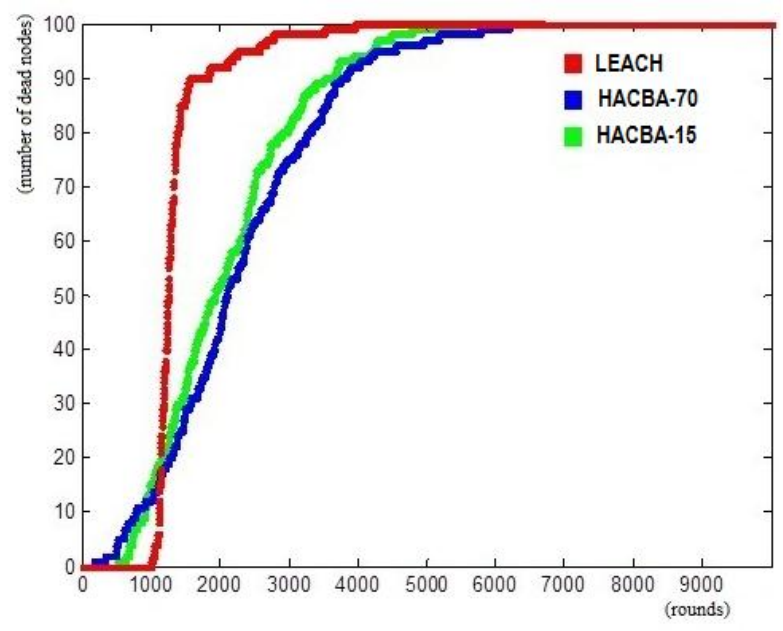

Fig. 7 Number of dead nodes against rounds

\section{CONCLUSION}

The HACBA protocol presents a strategy for dealing with the most important challenges in WSNs, energy consumption efficiency and message quality transmission, by integrating existing protocols.

HACBA represents a novel approach to the routing process in WSNs because it incorporates the hardware's characteristics. The routing process is adapted to the capabilities and limitations of the hardware.

Although the protocol was developed for forest fire monitoring, HACBA can be applied to other WSN applications.

Further works include sensor node creation in virtual platforms using the hardware description language SystemC and the application of artificial intelligence to increase sensor nodes' autonomy and energy optimization (for instance, use of genetic algorithm to establish access routes among sensor nodes in case of communication or operation failure).

\section{ACKNOWLEDGEMENTS}

The authors would like to thank the National Council of Scientific and Technological Development - CNPq and the National Institute of Science and Technology of Nano and Microeletronic Systems - INCT NAMITEC (Brazilian government).

\section{REFERENCES}

[1] Akyildiz, I. F., Vuran, M. C. 2010. Wireless Sensor Networks. 1.ed. John Wiley Publishing Company, 516p., ISBN: 978-0470-03601-3.

[2] Zheng, J., Jamalipour, A. 2009. Wireless Sensor Networks: A Networking Perspective. 1.ed. John Wiley Publishing Company, 489p., ISBN: 978-0470-16763-2.

[3] Heinzelman, W., Chandrakasan, A. and Balakrishnan, H. 2002. An Application-Specific Protocol Architecture for Wireless Microsensor Networks. In: IEEE Transactions on Wireless Communications, Vol. 1, No. 4, October 2002, pp. 660-670.

[4] Singh,S.K.; Singh, M.P.; Singh, D.K. 2010. Routing Protocols in Wireless Sensor Networks - A Survey. In: International Journal of Computer Science and Engineering Survey (IJCSES), 2(1), 49-61p., DOI : 10.5121/ijcses.2010.1206. 
[5] Lee, S. H. et al. 2009. Wireless Sensor Network Design for Tactical Military Applications: Remote Large-Scale Environments. In: IEEE Conference on Military Communications (MILCOM), 1-7p.

[6] Lou, E., Hill, D.L., Raso, J. V. 2010. A Wireless Sensor Network System to Determine Biomechanics of Spinal Braces During Daily Living. In: Medical and Biological Engineering and Computing, 48(3), 235-243p., doi: 10.1007/s11517-010-0575-4.

[7] Zhang, B. and Li,G. 2009. Survey of network management protocols in wireless sensor network. In International Conference on E-Business and Information System Security, May 2009, pp. 1-5.

[8] Sleman, A., Moeller, R. 2008. Integration of Wireless Sensor Network Services Into Other Home and Industrial Networks Using Device Profile for Web Services (DPWS). In: The 3rd International Conference on Information and Communication Technologies: From Theory to Applications (ICTTA). 1-5p., ISBN: 978-14244-1751-3.

[9] Assous, N. et al. 2009. Wireless Sensors for Instrumented Machines: Propagation Study for Stationary Industrial Environments. In: IEEE. Computer Aided Modeling and Design of Communication Links and Networks (CAMAD), 1-5p.

[10] Chen, K. et al. 2006. A Probabilistic Energy-Eficient Routing (PEER) Scheme for Ad-hoc Sensor Networks. In: Proceedings of the 3rd Annual IEEE Communications Society on Sensor and Ad Hoc Communications and Networks (SECON), 964-970p.

[11] Stipanicev, D., Vuko, T., Bodrozic, L. 2007. Location Determination of Automatic Forest Fire Monitoring Stations based on AHP and GIS Data. In: The International Emergency Management Society (TIEMS).

[12] Zheng, J.; Jamalipour, A. 2009. Wireless Sensor Networks: A Networking Perspective. Wiley-IEEE Press, 489p., ISBN: 978-0-470-16763-2.

[13] Ruiz, L. B., Nogueira, J. M. S., Loureiro, A. A. 2003. MANNA: A Management Architecture for Wireless Sensor Network. In: IEEE Communications Magazine, 41 (2), 116-125p.
[14] Glaser, J. et al. 2009. Investigating power-reduction for a reconfigurable sensor interface. In: Proceedings of the Austrian National Conference on the Design of Integrated Circuits and Systems, Graz, Austria. v. 7.

[15] Hempstead, M.; Wei, G.; Brooks, D. 2009. An accelerator-based wireless sensor network processor in $130 \mathrm{~nm}$ cmos. In: Proceedings of the 2009 international conference on Compilers, architecture, and synthesis for embedded systems. [S.1.: s.n.], p. 215-222.

[16] Pavai, K.. 2009. Study of Routing Protocols in Wireless Sensor Networks. In: International Conference on Advances in Computing, Control, \& Telecommunication Technologies, 522-525p.

[17] Bhatti, S., Xu, J.. 2009. Survey of Target Tracking Protocols using Wireless Sensor Network. In: Fifth International Conference on Wireless and Mobile Communications.

[18] Abdelkader, M., Hamdi, M. and Boudriga, N. 2008 Voronoi-Based Sensor Network Engineering for Target Tracking Using Wireless Sensor Networks. In: New Technologies, Mobility and Security, 2008. NTMS '08. , vol., no., pp.1-5, 5-7.

[19] Stemm, M. and Katz, R. H.. 1997. Measuring and reducing energy consumption of network rfaces in handheld devices. In: IEICE Transaction on Communications, vol. E80-B, 8, Aug.1997, pp. 11251131.

[20] Bahl, P. and Padmanabhan, V. N. 2000. Radar: A inbuilding rf-based user location and tracking system. In: Proceedings IEEE INFOCOM'OO, vol. 2, Tel-Aviv, Israel, Mar. 2000, pp. 775-784.

[21] Doherty, L., Pister, K. S.and L. E. Ghaoui. 2001. Convex position estimation in wireless sensor networks. In: International Journal of Computer Science \& Engineering Survey (IJCSES) Vol.1, No.2, pp. 16551663.

[22] Bruck, J.; Gao, J; Jiang, A. A. 2009. Localization and routing in sensor networks by local angle information. In: Journal ACM Transactions on Sensor Networks (TOSN), 5 (1), DOI: 10.1145/1464420.1464427. 